\title{
Evaluation of Cholangiocarcinoma Risk and its Related Factors In Wetland Geographical Communities of Ubon Ratchathani, Thailand
}

\author{
Nopparat Songserm ${ }^{1 *}$, Somkiattiyos Woradet ${ }^{2}$, Onanong Bureelerd ${ }^{1}$, Pattaraporn \\ Charoenbut ${ }^{1}$
}

\begin{abstract}
Wetland geographical areas have a higher incidence of Opisthorchis viverrini-associated cholangiocarcinoma (CCA), confirmed by data from geographic information systems, than other areas. Behavioral data also indicate that people in these areas traditionally eat uncooked freshwater fish dishes, a vehicle for $O$. viverrini infection. The best approach to reducing CCA incidence is decreasing risk factors together with behavior alteration. Evaluation of CCA risk and its related factors are first needed for planning the prevention and control programs in the future. We therefore aimed to evaluate the CCA risk and explore its related factors among people in wetland communities of Ubon Ratchathani, Thailand. A cross-sectional study was conducted between July and August 2014. In total 906 participants, with informed consent, completed questionnaires. Overall risk of CCA was determined by multiplying odds ratios (ORs) of the risk factors for CCA from literature reviews. A mean score of 5.95 was applied as the cut-off point. Assessment of factors related to overall risk of CCA was accomplished using conditional logistic regression. Of all participants, $60.15 \%$ had a high level of the overall risk of CCA. Factors related to the overall risk of CCA were gender $(p<0.001)$, marital status $(p<0.001)$, perceived susceptibility $(p=0.043)$ and prevention behavior for $\mathrm{CCA}(\mathrm{p}<0.001)$. In conclusion, most participants in this community had a high level of overall risk of CCA. Therefore, integrated prevention and control programs continue to be urgently required.
\end{abstract}

Keywords: Cholangiocarcinoma - risk factors - wetland community - Ubon Ratchathani, Thailand

Asian Pac J Cancer Prev, 17 (4), 1811-1815

\section{Introduction}

The statistical data from geographic information system (GIS) reported that a higher prevalence rate of liver fluke (Opisthorchis viverrini; O. viverrini) infection was found in wetland geographical areas than other areas (Wattanayingcharoenchai et al., 2011). As same as the GIS data of Ubon Ratchathani Province in Northeast Thailand showed that most areas are wetland areas, which a prevalence rate of liver fluke infection from a survey in 2009 was $20 \%$ (Sithithaworn et al., 2012). The reason that why the people in the wetland community had a high rate of liver fluke infection. The answer is they are residents near a river body and have easy access to fisheries; so they also have traditional behaviors of eating uncooked freshwater fish dishes (Vatanasapt et al., 1990; Sripa et al., 2007; Grundy-Warr et al., 2012). This consumption behavior is a source of liver fluke infection resulting in developing bile duct cancer or cholangiocarcinoma (CCA)
(Songserm et al., 2012; Manwong et al., 2013).

CCA is the most common cancer in Thailand with the incidence (mean annual age-standardized incidence rate, ASR 2010-2012) of 33.9 in men and 12.9 in women per 100,000, respectively. Also, it is the leading cancer in Ubon Ratchathani with the ASR of 52.9 in men and 23.9 in women per 100,000 , respectively (Imsamran et al., 2015). There were several known risk factors for CCA from the literature reviews in Thailand which are sorted with respect to decreasing severity: The first is consumption of raw freshwater fish dishes (3 times a week) which had a higher risk 3.40 times than those who did not, followed by $O$. viverrini infection with a risk of 3.09 times, alcohol drinking ( 3 times a week) had a risk of 2.13 times (Manwong et al., 2013), praziquantel use increased risk by 1.84 times (Kamsa-ard et al., 2013), and consumption of nitrosamine-contaminated foodstuffs increased risk by 1.20 times (Songserm et al., 2012). The best idea for reducing the incidence of CCA in high risk

\footnotetext{
${ }^{I}$ Department of Community Health, Faculty of Public Health, Ubon Ratchathani Rajabhat University, Ubon Ratchathani Rajabhat ${ }^{2}$ Department of Public Health, Faculty of Health and Sports Science, Thaksin University, Phatthalung, Thailand *For correspondence: nopparat.s@ubru.ac.th
} 
areas is decreasing the risk factors together with behavior alteration. So, evaluation of CCA risk and its related factors are firstly needed to prevent and control it in the future. We therefore aimed to evaluate the risk of CCA and explore its related factors among people in the wetland geographical communities of Ubon Ratchathani, Thailand.

\section{Materials and Methods}

\section{Study design and data collection}

Details of the study design and ethical consideration have been published previously (Songserm et al., 2015). Briefly, a cross-sectional study was conducted between July and August 2014 in the wetland geographical communities in Muang District of Ubon Ratchathani, which are surrounded with the basins of the Chi, Se Bai, and Mun Rivers. The community residents of ages 30 to 69 were randomly recruited by inviting only one member per household to participate. Finally, 906 participants, with informed consent, were interviewed by trained interviewers and completed the questionnaires. Research tools, assessment of research tools and data collection have been described in our previous publication (Songserm et al., 2015).

\section{Statistical analysis}

Evaluation of overall CCA risk was analyzed by using odds ratios (ORs) of the risk factors for CCA from the literature reviews in the high incidence area of Thailand.A summary of the results is provided in Table 1. The overall risk of CCA was determined by multiplying the ORs of the risk factors for CCA from the Table $1(0=$ "no", 1 = "yes"). The mean scores at $<5.95$ and $\geq 5.95$ were the cut-off points.

To assess the related factors of the overall risk of CCA among people in the wetland geographical communities of Ubon Ratchathani, the ORs with $95 \%$ confidence intervals (95\% CIs) were estimated using conditional logistic regression. Independent variables were the sociodemographic data, knowledge on liver fluke infection and CCA, health belief on liver fluke infection and CCA, and preventing behavior for CCA. The dependent variable was the overall risk of CCA $(<5.95$ scores = "low risk", $\geq 5.95$ scores = "high risk"). A P-value $<0.05$ was considered statistically significant. All statistical analyses were performed with a statistical package, STATA version 10 (Stata, College Station, TX).

\section{Results}

Nine hundred and six participants were randomly selected, $36.3 \%$ were men, $63.7 \%$ were women, and the average age was $51.39 \pm 10.29$ years old, ranging from 30 to 69 . The prevalence of risk factors for CCA among people in wetland geographical communities of Ubon Ratchathani is shown in Table 2. 58.50\% of the participants were alcohol drinker. Of those, $14.90 \%$ had the high drink $\geq 2,399$ units of all alcoholic beverages per month. $8.17 \%$ have had a history of liver fluke infection. $30.57 \%$ had used the praziquantel for preventing liver fluke. $82.56 \%$ of all participants had consumed raw freshwater fish dishes. Of those, $1.55 \%$ had a daily consumption. 814 of 906 participants $(89.85 \%)$ had a consumption of nitrosaminecontaminated foodstuffs. Dramatically, there were $37.97 \%$ of those had a daily consumption.

With respect to the evaluation of the overall risk of CCA among people in the wetland geographical communities of Ubon Ratchathani, most of participants $(60.15 \%)$ had a high overall risk of CCA ( $\geq 5.95$ scores) (Figure 1).

According to the multivariate analysis in Table 3, factors related to the overall risk of CCA among people in the wetland geographical communities of Ubon Ratchathani were sex $(\mathrm{p}<0.001)$, marital status $(\mathrm{p}<0.001)$, perceived susceptibility $(\mathrm{p}=0.043)$ and preventing behavior for CCA $(\mathrm{p}<0.001)$. There were 3 issues to be protective factors. Firstly, there was a clear association with sex: females had a decreased association with the overall risk of CCA when compared with males $(\mathrm{OR}=0.13$, 95\% CI: 0.09-0.19). Secondly, participants with a high perceived susceptibility had a decreased risk of CCA (OR=0.72, 95\% CI: 0.52-0.99). Lastly, participants with high level of preventative behavior had lower risk of CCA than those with a low level. Conversely, married

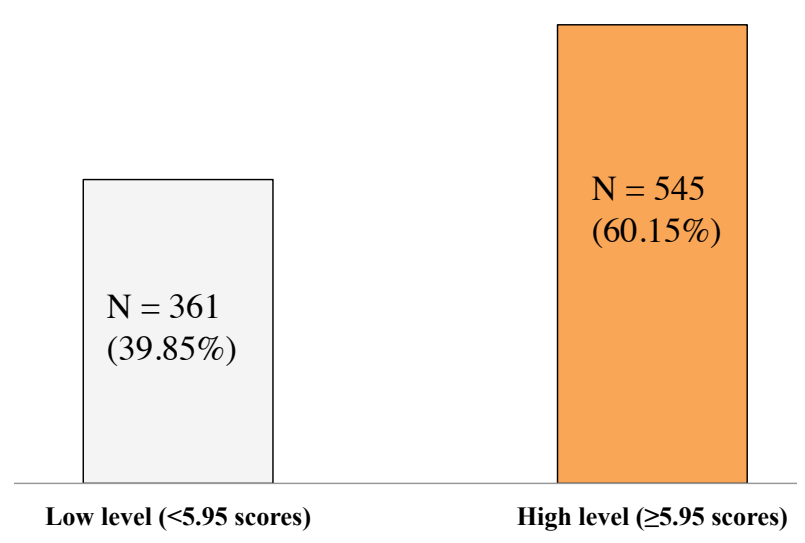

Figure 1. Overall Risk of Cholangiocarcinoma among Rural People in Wetland Geographical Communities of Ubon Ratchathani, Thailand

Table 1. Risk Factors and Odds Ratios of Cholangiocarcinoma from Literature Reviews in the High-Incidence Areas of Thailand

\begin{tabular}{|c|c|c|c|}
\hline Risk factors & Odds ratio $(\mathrm{OR})$ & Study sites & References \\
\hline Consumption of raw freshwater fish dishes (3 times/week) & 3.40 & Ubon Ratchathani & Manwong et al., 2013 \\
\hline Liver fluke infection (Anti-OV) & 3.09 & Ubon Ratchathani & Manwong et al., 2013 \\
\hline Alcohol consumption (3 times/week) & 2.13 & Ubon Ratchathani & Manwong et al., 2013 \\
\hline Praziquantel use & 1.84 & Khon Kaen & Kamsa-ard et al., 2013 \\
\hline Consumption of nitrosamine-contaminated foodstuffs & 1.20 & Khon Kaen & Songserm et al., 2012 \\
\hline
\end{tabular}


Table 2. Prevalence of Risk Factors for Cholangiocarcinoma among Rural People in Wetland Geographical Communities of Ubon Ratchathani, Thailand $(n=906)$

\begin{tabular}{lcc}
\hline Variables & Number & $\%$ \\
\hline Alcohol drinking & 376 & 41.50 \\
No & 530 & 58.50 \\
Yes & 376 & 41.50 \\
Units of alcohol per month of all alcohol drinking & \\
Non-drinker & 395 & 43.60 \\
$<2,399$ & 135 & 14.90 \\
$\geq 2,399$ & 376 & 41.50 \\
Age at which drinking started (all alcoholic beverages) \\
Non-drinker & 171 & 18.87 \\
$<18$ & 134 & 14.79 \\
$19-20$ & 151 & 16.67 \\
$21-30$ & 74 & 8.17 \\
$31+$ & $23.60 \pm 8.64$ \\
Mean \pm SD & $20(10: 60)$ \\
Median (Min : Max) & \multicolumn{2}{c}{} \\
History of liver fluke infection & 832 & 91.83 \\
No & 74 & 8.17 \\
Yes &
\end{tabular}

History of praziquantel use for preventing liver fluke

$\begin{array}{lrc}\text { Never used } & 629 & 69.43 \\ \text { Ever used } & 277 & 30.57 \\ \text { Average time of ever used } & 1.61 \pm 1.65(1: 11) \\ \quad \text { (Min:Max) } & \\ \text { Consumption of raw freshwater fish dishes } \\ \text { No } \\ \text { Yes } & 158 & 17.44 \\ & 748 & 82.56\end{array}$

Frequency of raw freshwater fish dishes consumption

$\begin{array}{lcc}\text { Non-consumer } & 158 & 17.44 \\ <1 / \text { month } & 588 & 64.90 \\ \text { Monthly } & 115 & 12.69 \\ \text { Weekly } & 31 & 3.42 \\ \text { Daily } & 14 & 1.55\end{array}$

Consumption of nitrosamine-contaminated foodstuffs

$\begin{array}{lcc}\text { No } & 92 & 10.15 \\ \text { Yes } & 814 & 89.85\end{array}$

Frequency of nitrosamine-contaminated foodstuffs consumption

\begin{tabular}{lcc} 
Non-consumer & 92 & 10.15 \\
$<1 /$ month & 206 & 22.74 \\
Monthly & 95 & 10.49 \\
Weekly & 169 & 18.65 \\
Daily & 344 & 37.97 \\
\hline
\end{tabular}

and widowed participants had a 2.60 times higher risk of CCA than unmarried participants.

\section{Discussion}

There were two objectives of this present study: (1) To evaluate the risk of CCA among people in the wetland geographical communities of Ubon Ratchathani, Thailand; the results show that $60.15 \%$ of all participants had a high overall risk of CCA ( $\geq 5.95$ scores). (2) To explore
Table 3. Multivariate Analysis of Factors Related to the Risk for Cholangiocarcinoma among Rural People in wetland Geographical Communities of Ubon Ratchathani, Thailand

\begin{tabular}{|c|c|c|c|c|}
\hline Variables & $\mathrm{OR}^{*}$ & $\mathrm{OR} * *$ & $95 \% \mathrm{CI}$ & p-value \\
\hline Sex & & & & $<0.001$ \\
\hline Male & 1.00 & 1.00 & & \\
\hline Female & 0.13 & 0.13 & $0.09-0.19$ & \\
\hline Age (years) & & & & 0.178 \\
\hline$\leq 44$ & 1.00 & 1.00 & & \\
\hline $45-59$ & 0.99 & 0.84 & $0.57-1.25$ & \\
\hline$\geq 60$ & 0.75 & 0.63 & $0.39-1.03$ & \\
\hline Occupation & & & & 0.053 \\
\hline Farmer/Laborer & 1.00 & 1.00 & & \\
\hline Commercial/Trading & 0.57 & 0.45 & $0.22-0.93$ & \\
\hline Government officer & 1.15 & 1.30 & $0.51-3.35$ & \\
\hline Education level & & & & 0.122 \\
\hline Primary school & 1.00 & 1.00 & & \\
\hline Secondary school & 1.18 & 0.72 & $0.46-1.12$ & \\
\hline $\begin{array}{l}\text { Bacheler degree or } \\
\text { higher }\end{array}$ & 0.61 & 0.41 & $0.16-1.11$ & \\
\hline Marital status & & & & $<0.001$ \\
\hline Single & 1.00 & 1.00 & & \\
\hline Married & 2.23 & 2.61 & $1.51-4.52$ & \\
\hline Divorced & 0.78 & 1.19 & $0.57-2.51$ & \\
\hline Widowed & 1.71 & 2.66 & $1.04-6.80$ & \\
\hline \multicolumn{4}{|c|}{ Household income per year (Baht) } & 0.240 \\
\hline$\leq 10,000$ & 1.00 & 1.00 & & \\
\hline $10,001-15,000$ & 1.45 & 1.50 & $0.91-2.47$ & \\
\hline \multirow[t]{2}{*}{$>15,000$} & 1.30 & 1.30 & $0.88-1.91$ & \\
\hline & & $\mathrm{CCA}$ & & \\
\hline \multicolumn{4}{|c|}{$\begin{array}{l}\text { Knowledge on opisthorchiasis and } \\
\text { cholangiocarcinoma }\end{array}$} & 0.067 \\
\hline Low ( $\leq 7$ scores $)$ & 1.00 & 1.00 & & \\
\hline High (>7 scores) & 0.77 & 0.74 & $0.54-1.02$ & \\
\hline \multicolumn{4}{|l|}{ Perceived susceptibility } & 0.043 \\
\hline Low ( $\leq 11.72$ scores) & 1.00 & 1.00 & & \\
\hline High (>11.72 scores) & 0.73 & 0.72 & $0.52-0.99$ & \\
\hline \multicolumn{4}{|l|}{ Perceived seriousness } & 0.226 \\
\hline Low ( $\leq 11.93$ scores) & 1.00 & 1.00 & & \\
\hline High (>11.93 scores) & 1.27 & 1.23 & $0.88-1.72$ & \\
\hline \multicolumn{4}{|l|}{ Perceived benefits } & 0.605 \\
\hline Low ( $\leq 12.72$ scores) & 1.00 & 1.00 & & \\
\hline High (>12.72 scores) & 0.99 & 1.13 & $0.72-1.77$ & \\
\hline \multicolumn{4}{|l|}{ Perceived barriers } & 0.581 \\
\hline Low ( $\leq 12.13$ scores) & 1.00 & 1.00 & & \\
\hline High (>12.13 scores) & 0.89 & 0.91 & $0.67-1.26$ & \\
\hline \multicolumn{4}{|c|}{ Preventing behavior for CCA } & $<0.001$ \\
\hline Low ( $\leq 22$ scores $)$ & 1.00 & 1.00 & & \\
\hline High (>22 scores) & 0.38 & 0.51 & $0.37-0.70$ & \\
\hline \multicolumn{4}{|c|}{ Community participation for CCA prevention } & 0.348 \\
\hline Low ( $\leq 18$ scores) & 1.00 & 1.00 & & \\
\hline High ( $>18$ scores $)$ & 0.98 & 1.17 & $0.84-1.62$ & \\
\hline
\end{tabular}

its related factors; it was found that sex, marital status, perceived susceptibility and preventative behavior for CCA were factors related to the overall risk of CCA.

Prevalence of raw freshwater fish consumption (KoiPla) among people in the wetland communities of Ubon Ratchathani was $82.56 \%$. Of those, 64.13 had consumed cyprinoid fish - a direct vehicle of liver fluke infection. Moreover, $73.95 \%$ of all participants had consumed 
dishes containing raw- and/or semi-raw freshwater fish (Pla-Chom, Pla-Choa). $89.85 \%$ of all participants had consumed nitrosamine-contaminated foodstuffs (Pla-ra). Indeed, consumption of raw freshwater (cyprinoid) fish and nitrosamine-contaminated foodstuffs is associated with increased the risk of CCA. It is associated with a hospital-based case-control study in Ubon Ratchathani, which reported that consumption of raw freshwater fish dishes (3 times a week) increased the risk of CCA by 3.40 times when compared with the participants who did not (Manwong et al., 2013). Also, a nested case-control study within the Khon Kaen Cohort Study reported that consumption of nitrosamine-contaminated foodstuffs had increased the risk of CCA at 1.20 times. However, it is based on dose-response effect (Songserm et al., 2012).

From the survey of liver fluke infection among people in the wetland communities of Ubon Ratchathani, it was found that $8.17 \%$ have had a history of liver fluke infection. Indeed, the International Agency for Research on Cancer (IARC) concluded in 1994 that O. viverrini infection was sufficient evidence as a bio-carcinogenic risk factor for CCA in humans (IARC, 1994), and then there were several subsequent studies that have confirmed this evidence conclusion (Sriamporn et al., 2004; Honjo et al., 2005; Poomphakwaen et al., 2009; Songserm et al., 2011; Manwong et al., 2013).

In this present study, the overall prevalence of alcohol consumption among people in the wetland communities of Ubon Ratchathani was $58.50 \%$. Most of them drank a beer $(46.80 \%)$, followed by white whisky $(36.75 \%)$ and red whisky (25.28\%), respectively. Appallingly, the youngest participants who were alcohol drinkers were 10 years of age. Actually, alcohol drinking is one of main risk factors for CCA from several studies in Thailand (Honjo et al., 2005; Poomphakwaen et al., 2009; Songserm et al., 2012; Manwong et al., 2013), and elsewhere (Shin et al., 1996; Chalasani et al., 2000). It is possible that ethanol from alcoholic beverages expands the effect of excreting carcinogens from nitrosamine-contaminated foodstuffs, resulting in more inflammation and CCA progression (Honjo et al., 2005).

With respect to the praziquantel use among people in the wetland communities of Ubon Ratchathani, the results showed that $30.57 \%$ had used the praziquantel for preventing the liver fluke. Of those, some participants had used it up to 11 times. From the past literature reviews, praziquantel used was a risk factor for CCA (Elkins et al., 1996; Honjo et al., 2005). It is reasonable that participants who have frequently used the praziquantel have also regularly consumed the dishes of raw freshwater fish, resulting in more re-infection, more inflammation and more induction of CCA.

This present study showed that the related factors of the overall risk of CCA were sex, marital status, perceived susceptibility and preventative behavior for CCA. It is associated with a cross-sectional study in Khon Kaen and Mahasarakham Provinces in Northeast Thailand. The results showed that males associated with an increased risk of CCA when compared with females (adjusted $\mathrm{OR}=3.00$; 95\% CI: 0.8-11.2) (Haswell et al., 1994).

In conclusion, the best way for CCA control in wetland geographical areas, which is a high incidence area of CCA, is primary prevention. The primary prevention of CCA is based on efforts to reduce the risk factors together with behavior alteration. Therefore, evaluation of CCA risk and its related factors in high incidence areas are firstly needed to estimate a situation for planning the prevention and control program in the future. In addition, most of all participants in this community had a high level of the overall risk of CCA. Therefore, integrated prevention and control programs are urgently required.

\section{Acknowledgements}

This research was supported by the Thailand Research Fund and Ubon Ratchathani Rajabhat University (Research grant No. 2557A16402006).

\section{References}

Chalasani N, Baluyut A, Ismail A, et al (2000). Cholangiocarcinoma in patients with primary sclerosing cholangitis: a multicenter case-control study. Hepatol 31, 7-11.

Elkins DB, Mairiang E, Sithithaworn P, et al (1996). Crosssectional patterns of hepatobiliary abnormalities and possible precursor conditions of cholangiocarcinoma associated with Opisthorchis viverrini infection in humans. Am J Trop Med Hyg, 55, 295-301.

Grundy-Warr C, Andrews RH, SithithawornP, et al (2012). Raw attitudes, wetland cultures, life-styles: Socio-cultural dynamics relating to Opisthorchis viverrini in the Mekong basin. Parasitol Int, 61, 65-70.

Haswell-Elkins MR, Mairiang E, Mairiang P, et al (1994). Cross-sectional study of Opisthorchis viverrini infection and cholangiocarcinoma in communities within a high-risk area in northeast Thailand. Int J Cancer, 59, 505-9.

Honjo S, Srivatanakul P, Sriplung H, et al (2005). Genetic and environmental determinants of risk for cholangiocarcinoma via Opisthorchis viverrini in a densely infested area in Nakhon Phanom, northeast Thailand. Int J Cancer, 117, 854-60.

IARC (1994). Schistosomes, liver flukes and Helicobacter pylori. IARC Monogr Eval Carcinog Risks Hum, 61, 1-241.

Imsamran W, Chaiwerawattana A, Wiangnon S, et al. Cancer in Thailand 2010-2012, vol. VIII. Bangkok: Bangkok Medical Publisher; 2015.

Kamsa-ard S, Laopaiboon M, Luvira V, et al (2013). Association between praziquantel and cholangiocarcinoma in patients infected with Opisthorchis viverrini: a systematic review and meta-analysis. Asian Pac J Cancer Prev, 14, 7011-6.

Manwong M, Songserm N, Promthet S, et al (2013). Risk factors for cholangiocarcinoma in the lower part of Northeast Thailand: a hospital-based case-control study. Asian Pac J Cancer Prev, 14, 5953-6.

Poomphakwaen K, Promthet S, Kamsa-Ard S, et al (2009). Risk factors for cholangiocarcinoma in Khon Kaen, Thailand: a nested case- control study. Asian Pac J Cancer Prev, 10, 251-8.

Shin HR, Lee CU, Park HJ, et al (1996). Hepatitis B and C virus, Clonorchis sinensis for the risk of liver cancer: a case-control study in Pusan, Korea. Int J Epidemiol, 25, 933-40.

Sithithaworn P, Andrews RH, Van De N, et al (2012). The current status of opisthorchiasis and clonorchiasis in the Mekong Basin. Parasitol Int, 61, 10-6.

Songserm N, Bureelerd O, Thongprung S, et al (2005). Community participation in cholangiocarcinoma prevention 
in Ubon Ratchathani, Thailand: relations with age and health behavior. Asian Pac J Cancer Prev, 16, 7375-9.

Songserm N, Promthet S, Sithithaworn P, et al (2011). MTHFR polymorphisms and Opisthorchis viverrini infection: a relationship with increased susceptibility to cholangiocarcinoma in Thailand. Asian Pac J Cancer Prev, 12, 1341-5.

Songserm N, Promthet S, Sithithaworn P, et al (2012). Risk factors for cholangiocarcinoma in high-risk area of Thailand: role of lifestyle, diet and methylenetetrahydrofolate reductase polymorphisms. Cancer Epidemiol, 36, 89-94.

Sriamporn S, Pisani P, Pipitgool V, et al (2004). Prevalence of Opisthorchis viverrini infection and incidence of cholangiocarcinoma in Khon Kaen, Northeast Thailand. Trop Med Int Health, 9, 588-94.

Sripa B, Kaewkes S, Sithithaworn P, et al (2007). Liver fluke induces cholangiocarcinoma. PLoS Med, 4, 201.

Vatanasapt V, Uttaravichien T, Mairiang E, et al (1990). Cholangiocarcinoma in north-east Thailand. Lancet, 335, 116-7.

Wattanayingcharoenchai S, Nithikathkul C, Wonsaroj T, et al (2011). Geographic information system of Opisthorchis viverrini in northeast Thailand. Asian Biomedicine, 5, 687-91. 